\title{
Attractor Metadynamics in Adapting Neural Networks
}

\author{
Claudius Gros, Mathias Linkerhand, Valentin Walther \\ Institute for Theoretical Physics, Goethe University Frankfurt, Germany \\ \{gros07, linkerhand, walther\}@itp. uni-frankfurt.de \\ http://itp.uni-frankfurt.de/ gros
}

\begin{abstract}
Slow adaption processes, like synaptic and intrinsic plasticity, abound in the brain and shape the landscape for the neural dynamics occurring on substantially faster timescales. At any given time the network is characterized by a set of internal parameters, which are adapting continuously, albeit slowly. This set of parameters defines the number and the location of the respective adiabatic attractors. The slow evolution of network parameters hence induces an evolving attractor landscape, a process which we term attractor metadynamics. We study the nature of the metadynamics of the attractor landscape for several continuoustime autonomous model networks. We find both first- and second-order changes in the location of adiabatic attractors and argue that the study of the continuously evolving attractor landscape constitutes a powerful tool for understanding the overall development of the neural dynamics.
\end{abstract}

Keywords: adiabatic attractors, attractor metadynamics, neural networks, adaption, homeostasis

\section{Fast Neural Dynamics vs. Slow Adaption Processes}

Complex dynamical systems are often characterized by a variety of timescales and the brain is no exception here 112. It has been observed that the neural dynamics is contingent, for time scales ranging from hundreds of milliseconds to minutes, on the underlying anatomical network structure in distinct ways 3 . This relation between anatomy and the timescale characterizing neural activity is present even for autonomous systems, viz in the absence of external stimuli. It has been proposed, complementarily, that certain temporal aspects of the brain activity may reflect the multitude of timescales present in the environment 4], and could be induced through adaptive processes $[5$.

The neurons in the brain are faced with the problem, in a related perspective, of maintaining long-term functional stability on both the single neuron level, as well as on the level of network activities, in view of the fact that the constituents of the molecular and biochemical machinery, such as ion channel proteins and synaptic receptors, have lifetimes ranging only from minutes to weeks [6]. This situation results in the need to regulate homeostatically both the inter-neural 
synaptic strength [7, and the intra-neural parameters, the latter process termed intrinsic plasticity 89 .

Homeostatic mechanisms in the brain can be regarded as part of the generic control problem of the overall brain dynamics [10, with the adaption of neural parameters being necessary to achieve certain targets [11. Here we study the consequences of ongoing slow adaption for the time evolution of the landscape of adiabatic attractors, viz of the attractors of the dynamical system obtained by temporarily freezing the adaption process. We find that the locus of the instantaneous attracting state guides the overall time evolution and that the study of the attractor metadynamics, which find to be possibly both continuous and discontinuous, constitutes a powerful tool for the study of evolving neural networks.

\section{Adapting Continuous-Time Recurrent Neural Networks}

We consider continuous-time neural networks [1213], defined by

$$
\dot{x}_{i}=-\Gamma x_{i}+\sum_{j} w_{i j} y_{j}, \quad y_{i}=\frac{1}{1+\mathrm{e}^{a_{i}\left(b_{i}-x_{i}\right)}} .
$$

One may consider either the firing rates $y_{i}=y_{i}(t)$ as the primary dynamical variables or, equivalently, the corresponding membrane potentials $x_{i}=x_{i}(t)$, with the sigmoidal $g(z)=1 /(1+\exp (z))$ constituting the standard non-linear input-output relation for a single neuron. One denotes $a_{i}$ the gain (slope) of the sigmoidal and $b_{i}$ the respective threshold. Here $\Gamma>0$ sets the relaxation rate for the membrane potentials $x_{i}$ and the $w_{i j}$ are the inter-neural synaptic weights.

One speaks of intrinsic adaption when the internal parameters of an individual neuron adapt slowly over time [1415. In our case the bias $a_{i}$ and threshold $b_{i}$. This kind of internal adaption is necessary for keeping the output $y_{i}(t) \in[0,1]$ within the desired dynamical range, viz within the working regime of the dynamical system. Anatomical constraints such as the limited availability of energy are imposed on the long-term firing statistics of each neuron. On a functional level, the firing patterns are expected to encode maximal information. This distribution of maximal information is at the same time the least biased or 'noncomittal' with respect to the constraints [16] and it is obtained by maximizing Shannon's information entropy. Given a certain mean $\mu$, here the mean target firing rate [2], the desired output distribution is an exponential,

$$
p_{\lambda}(y) \propto \mathrm{e}^{\lambda y}, \quad \mu=\int d y y p(y),
$$

for the neural model (1), with $\lambda$ being the respective Langrange multiplier. The distance of a time series of data, like the neural firing rate $y_{i}(t)$ for a given neuron $i$, relative to this target distribution function $p_{\lambda}(y)$ is captured by the Kullback-Leibler divergence $K_{i}[2$

$$
K_{i}=\int d y p_{i}(y) \log \left(\frac{p_{i}(y)}{p_{\lambda}(y)}\right), \quad p_{i}(y)=\lim _{T \rightarrow \infty} \int_{0}^{T} \delta\left(y-y_{i}(t-\tau)\right) \frac{d \tau}{T},
$$




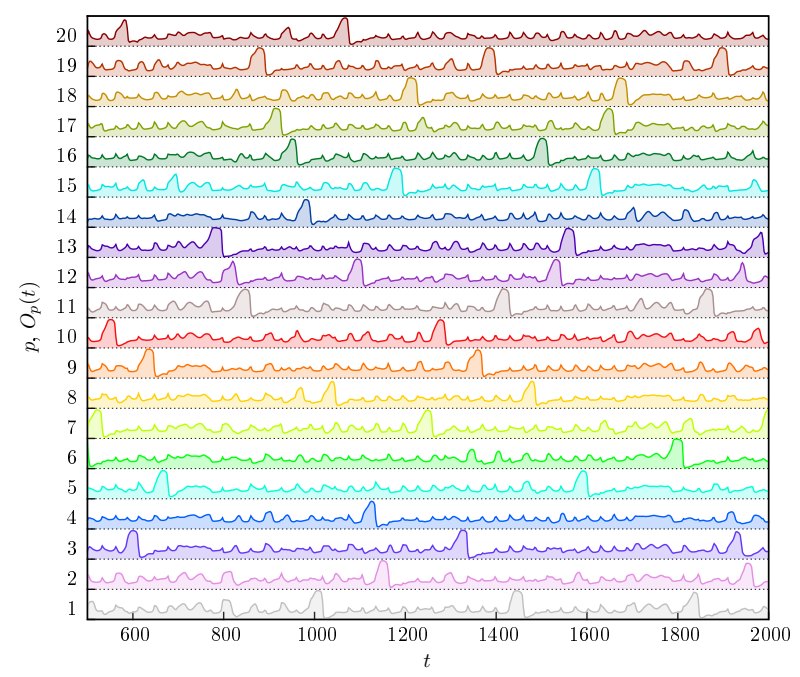

Fig. 1. A network with $N=1000$ neurons and $N_{p}=20$ encoded attractor states, see Eq. (5). The neurons adapt, trying to optimized the relative information content (3) of their respective activities. Shown are vertically displaced, as a function of time $t$, time lines of the overlaps $O_{p}(t) \in[0,1]$, see Eq. (6). Notice, that the polyhomeostatic adaption (4) leads to transient-state dynamics, one attractor relict $\xi^{p}$ after the other is transiently visited by the state of network activities, as evident by the bumps in the respective time lines.

where $p_{i}(y)$ is the time-averaged distribution of $y_{i}(t)$. One can now optimize the adaption by minimizing (3) with respect to the intrinsic parameters $a_{i}$ and $b_{i}$ and one obtains 17,18]

$$
\begin{array}{ll}
\dot{a}_{i}=\epsilon_{a}\left(1 / a_{i}+\left(x_{i}-b_{i}\right) \theta\right) & \\
\dot{b}_{i}=\epsilon_{b}\left(-a_{i}\right) \theta, & \theta=1-2 y_{i}+\lambda\left(1-y_{i}\right) y_{i}
\end{array}
$$

with $\epsilon_{a}$ and $\epsilon_{b}$ being adaption rates for the gain $a_{i}$ and the threshold $b_{i}$ respectively. In effect, the system is given an entire distribution function $p_{\lambda}(y)$ as an adaption target. The adaption rules (4) hence generalize the principle of homeostasis, which deals with regulating a single scalar quantity, and have been denoted polyhomeostatic optimization [19].

\section{Transient State Dynamics}

A convenient way to construct networks with a predefined set $\xi^{p}=\left(\xi_{1}^{p}, \xi_{2}^{p}, ..\right)$ of attracting states, with $p=1, . ., N_{p}$, is by selecting the synaptic weights as [20]

$$
w_{i j} \propto \sum_{p}\left(\xi_{i}^{p}-\bar{\xi}_{i}\right)\left(\xi_{j}^{p}-\bar{\xi}_{j}\right),
$$



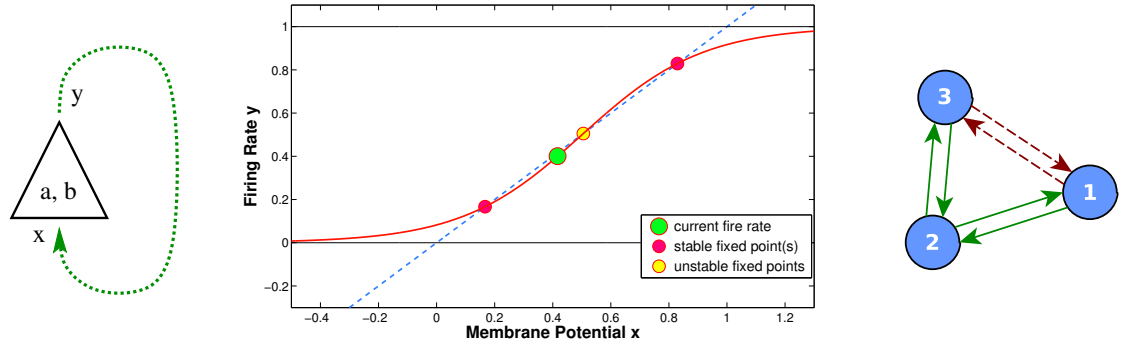

Fig. 2. Left: The autapse, a neural net with a single, self-coupled neuron. The output is directly fed to the input with $w_{11}=1$. Middle: Depending on the values of the intrinsic parameters $a$ and $b$ there may be one or two stable fixpoints $\dot{x}=0$ for the autapse, and one unstable fixpoint. The number and the position of the adiabatic fixpoints change when the gain $a=a(t)$ and the threshold $b=b(t)$ slowly adapt through (4). Shown are $y(x)$ (red solid line) and $x$ (dashed black line), compare Eq. (1). Right: A three-site network with inhibitory (red) and excitatory (green) synaptic weights.

where $\bar{\xi}_{j}$ is a local activity, averaged over all encoded patterns $\xi^{p}$. With the Hopfield encoding (5) one can hence construct attractor networks having point attractors close to the patterns $\xi^{p}$, with a given, predefined average activity level $\bar{\xi}^{p}=\sum_{i} \xi_{i}^{p} / N$, where $N$ is the number of neurons in the network.

As a first application we study a network of $N=1000$ neurons with the synaptic weights selected using the Hopfield encoding (5) and $N_{p}=20$ random binary patterns $\xi^{p}=\left(\xi_{1}^{p}, . ., \xi_{N}^{p}\right)$ drawn from an uniform distribution. We define with

$$
O_{p}(t)=\frac{\sum_{i} \xi_{i}^{p} y_{i}}{\left\|\xi^{p}\right\|\|y\|}, \quad \quad\|z\| \equiv \sqrt{\sum_{i} z_{i}^{2}}
$$

the overlap between the current state $y(t)=\left(y_{1}(t), . ., y_{N}(t)\right)$ and a given stored attractor state $\xi^{p}$, in terms of the respective normalized scalar product.

In Fig. 1 we show a typical simulation result for the overlaps $O_{p}(t) \in[0,1]$, with the individual time lines being shown vertically displaced and color-coded. The parameters used for the simulation are $\Gamma=1, \epsilon_{a}=0.1, \epsilon_{b}=0.01$ and $\bar{\xi}^{p}=0.2, \mu=0.2$. Alternative values for the adaption rates $\epsilon_{a, b}$ lead qualitatively to similar behaviors, whenever the adaption process is substantially slower than the neural dynamics (1). One observes two distinct features.

- For $\epsilon_{a}=\epsilon_{b}=0$ the dynamics would eventually settle into a steady state close to one of the stored patterns $\xi^{p}$. The dynamical activity is, on the other hand, continuous and autonomously ongoing when intrinsic adaption is present, as evident from Fig. 1. This is due to the fact that the system tries to achieve exponentially distributed firing-rate distributions. Without adaption the individual $p_{i}(y)$ would be simple $\delta$-functions in any fixpoint state and this would lead to a very high and therefore sub-optimal Kullback-Leibler divergence (3). 

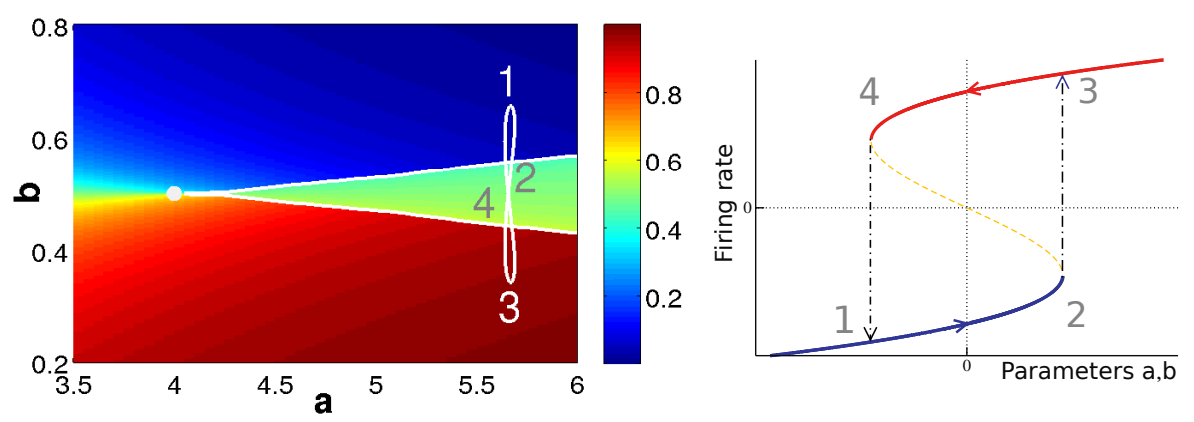

Fig. 3. Left: Phase diagram of the autapse, as illustrated in Fig. 2 The activity $y \in[0,1]$ of the fixpoint is color-coded, for fixed gains $a$ and thresholds $b$ of the sigmoidal, see Eq. (1), when only a single stable fixpoint is present. The greenish area within the two white lines denotes the phase space containing two stable fixpoints. The thick white line is the limiting cycle for polyhomeostatically adapting intrinsic parameters $(a(t), b(t))$, compare Eq. (4). Right: The firing rate of the adiabatic fixpoint (stable/unstable: thick/dashed lines) as function of the intrinsic parameters. The arrows and numbers indicate the section of the hysteresis loop in the landscape of adiabatic fixpoints corresponding to the equally labeled sections of the limiting cycle of $(a(t), b(t))$ shown in the left panel.

- The overlaps $O_{p}(t)$ are, most of the time, relatively small with temporally well defined characteristic bumps corresponding to dynamical states $y(t)$ approaching closely one of the initially stored patterns $\xi^{p}$. This type of dynamics has been termed transient-state [21] and latching [22] dynamics and may be used for semantic learning in autonomously active neural networks [23]24].

The inclusion of intrinsic adaption hence destroys all previously present attracting states. When the adaption process is slow and hence weak, with the actual values for the adaption rates $\epsilon_{a}$ and $\epsilon_{b}$ not being relevant, the system will however still notice the remains of the original point attractors and slow down when close by. The resulting type of network has been termed attractor relict network 23.

\section{Discontinuous Attractor Metadynamics}

A complete listing of all attracting states and the study of their respective time evolution is cumbersome for a large network like the one of Fig. 1. For an indepth study we have selected two small model systems, we start with a single, self-coupled neuron, the autapse, as illustrated in Fig. 2 (left).

The fixpoint condition is $x=y(x)$, for $\Gamma=1=w_{11}$, and it is depicted in Fig. 2 (middle). Depending on the location of the turning point $b$ of the sigmoidal and on its steepness $a$, there may be either one or two stable fixpoints, the 

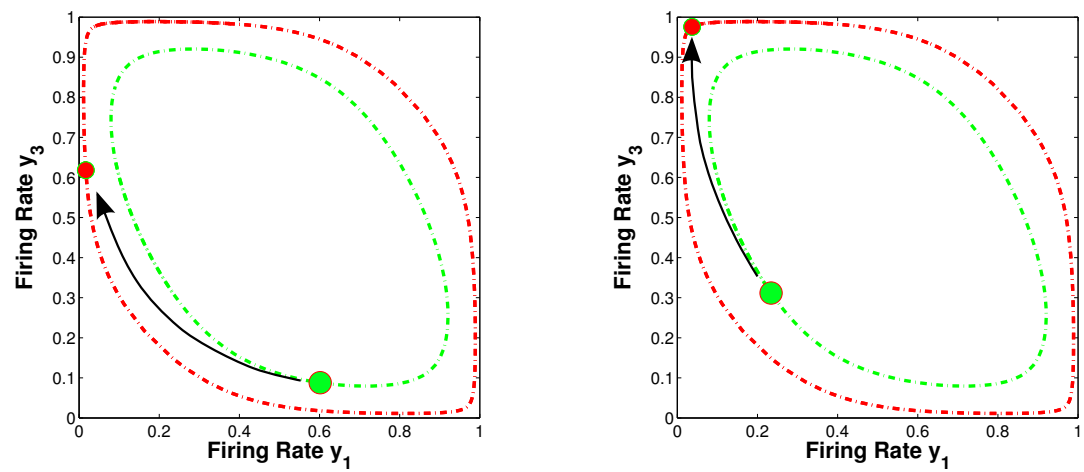

Fig. 4. For the three site network illustrated in Fig. 2 (right), the time evolution of the firing rates $\left(y_{1}(t), y_{3}(t)\right)$. The green line is the trajectory of the final limiting cycle and the red line of the single adiabatic attractor present in the system. The black arrows illustrate the instantaneous flow, attracting the current dynamical state (green filled circles) to the current position of the adiabatic attractor (red filled circles). The right panel follows in time shortly after the left panel.

respective phase diagram is presented in Fig. 3 (left). Additionally an unstable fixpoint may be present (central region).

The actual values $(a(t), b(t))$ of the intrinsic parameters polyhomeostatically adapt via (4), an example of an actual state is included in Fig. 2 (middle) and the final limiting cycle in Fig. 3 (left). The internal parameters settle, after an initial transient, in a region of phase space crossing two first-order phase transitions at which the number of attractors changes from $1 \leftrightarrow 2 \leftrightarrow 1$, resulting in a hysteresis loop for the adiabatic attractor landscape, compare Fig. 3 (right).

In the limit of long times the internal parameters, as given by the white elongated eight-shaped loop in Fig. 3. (left), stay for finite time intervals in the regions of the phase diagram characterized by a single fixpoint (top/bottom : blue/red). The limiting cycle of the adaption trajectory hence overshoots the hysteresis loop characterized by the vertical transitions illustrated in Fig. 3 (right).

The dynamics is relatively slow on the hysteresis branches $1 \rightarrow 2$ and $3 \rightarrow$ 4 and becomes very fast when the local adiabatic fixpoints vanishes. At this point the system is forced to rapidly evolve towards the opposite branch of the hysteresis loop, an example of self-organized slow-fast dynamics.

\section{Continuous Attractor Metadynamics}

As a second model system we consider the three-site network depicted in Fig. 2 (right), with $w_{12}=w_{21}=1=w_{32}=w_{23}$ and $w_{31}=w_{13}=-1$. At first sight one may expect an attractor metadynamics equivalent to the one of the autapse, since the three-site net also has two possible attracting states $\left(y_{1}^{*}, y_{2}^{*}, y_{3}^{*}\right)$, with either $y_{2}^{*}$ and $y_{1}^{*}$ large and $y_{3}^{*}$ small, or with $y_{2}^{*}$ and $y_{3}^{*}$ large and $y_{1}^{*}$ small. 
There is indeed a region in phase space for which these two fixpoints coexist [25], but the system adapts the six internal parameters $a_{i}(t)$ and $b_{i}(t)$ such that a single adiabatic fixpoint remains, which morphs continuously under the influence of the polyhomeostatic adaption (4).

In Fig. 4 we present the resulting limiting cycle of the full dynamics projected onto the $\left(y_{1}, y_{3}\right)$ plane (the activity of $y_{2}$ is intermediate and only weakly changing). One observes that the adiabatic fixpoint moves on a continuous trajectory, an adiabatic limiting cycle. This behavior contrasts with the time evolution of the attractor landscape observed for the autapse, as presented in Fig. 3 (right), which is characterized by a discontinuous hysteresis loop.

The adiabatic fixpoint approaches $\left(y_{1}^{*} \approx 1, y_{3}^{*} \approx 0\right)$ and $\left(y_{1}^{*} \approx 0, y_{3}^{*} \approx 1\right)$ repeatedly, as evident in Fig. 4. The corresponding phase space trajectory then slows down, as one can observe when plotting the actual time evolution, an example of transient-state dynamics.

\section{Carrot and Donkey Dynamics}

In the metaphor of the donkey trying to reach the carrot it carries itself, the animal will never reach its target. The case of self-generated attractor metadynamics studied here is analogous. The current dynamical state is attracted by the nearest adiabatic attractor, but the systems itself morphs the attractor continuously when the trajectory tries to close in. The locus of the attractor evolves, either continuously or discontinuously, and the trajectory is then attracted by the adiabatic fixpoint at its new locus.

This feature allows to characterize decision processes in the brain and in model task problems dynamically [2627, and choice options can be extracted in terms of corresponding adiabatic fixpoints. Here we studied autonomous systems, starting from attractor networks, with the aim to obtain a first overview regarding the possible types of self-generated attractor metadynamics.

\section{Acknowledgments}

The authors would like to thank Peter Hirschfeld for illuminating suggestions.

\section{References}

1. Izhikevich, E.M.: Dynamical systems in neuroscience. The MIT press (2007)

2. Gros, C.: Complex and adaptive dynamical systems: A primer. Springer Verlag (2013)

3. Honey, C.J., Kötter, R., Breakspear, M., Sporns, O.: Network structure of cerebral cortex shapes functional connectivity on multiple time scales. Proceedings of the National Academy of Sciences 104(24) (2007) 10240-10245

4. Kiebel, S.J., Daunizeau, J., Friston, K.J.: A hierarchy of time-scales and the brain. PLoS Computational Biology 4(11) (2008) e1000209 
5. Ulanovsky, N., Las, L., Farkas, D., Nelken, I.: Multiple time scales of adaptation in auditory cortex neurons. The Journal of Neuroscience 24(46) (2004) 10440-10453

6. Marder, E., Goaillard, J.M.: Variability, compensation and homeostasis in neuron and network function. Nature Reviews Neuroscience 7(7) (2006) 563-574

7. Turrigiano, G.G., Nelson, S.B.: Homeostatic plasticity in the developing nervous system. Nature Reviews Neuroscience 5(2) (2004) 97-107

8. Daoudal, G., Debanne, D.: Long-term plasticity of intrinsic excitability: learning rules and mechanisms. Learning \& Memory 10(6) (2003) 456-465

9. Echegoyen, J., Neu, A., Graber, K.D., Soltesz, I.: Homeostatic plasticity studied using in vivo hippocampal activity-blockade: synaptic scaling, intrinsic plasticity and age-dependence. PloS one 2(8) (2007) e700

10. O'Leary, T., Wyllie, D.J.: Neuronal homeostasis: time for a change? The Journal of physiology $\mathbf{5 8 9}(20)$ (2011) 4811-4826

11. Ge, S., Hang, C.C., Lee, T.H., Zhang, T.: Stable adaptive neural network control. Springer Publishing Company, Incorporated (2010)

12. Beer, R.D.: On the dynamics of small continuous-time recurrent neural networks. Adaptive Behavior 3(4) (1995) 469-509

13. Beer, R.D., Gallagher, J.C.: Evolving dynamical neural networks for adaptive behavior. Adaptive behavior 1(1) (1992) 91-122

14. Triesch, J.: A gradient rule for the plasticity of a neuron's intrinsic excitability. Artificial Neural Networks: Biological Inspirations-ICANN 2005 (2005) 65-70

15. Marković, D., Gros, C.: Intrinsic adaptation in autonomous recurrent neural networks. Neural Computation 24(2) (2012) 523-540

16. Jaynes, E.T.: Information theory and statistical mechanics. Physical review 106(4) (1957) 620

17. Linkerhand, M., Gros, C.: Self-organized stochastic tipping in slow-fast dynamical systems. Mathematics and Mechanics of Complex Systems 1(2) (2013) 129-147

18. Steil, J.J.: Online reservoir adaptation by intrinsic plasticity for backpropagationdecorrelation and echo state learning. Neural Networks 20(3) (2007) 353-364

19. Markovic, D., Gros, C.: Self-Organized Chaos through Polyhomeostatic Optimization. Physical Review Letters 105(6) (August 2010)

20. Hopfield, J.J.: Neural networks and physical systems with emergent collective computational abilities. Proceedings of the national academy of sciences $\mathbf{7 9}(8)$ (1982) 2554-2558

21. Gros, C.: Neural networks with transient state dynamics. New Journal of Physics 9(4) (2007) 109

22. Russo, E., Namboodiri, V.M., Treves, A., Kropff, E.: Free association transitions in models of cortical latching dynamics. New Journal of Physics 10(1) (2008) 015008

23. Gros, C.: Cognitive computation with autonomously active neural networks: an emerging field. Cognitive Computation 1(1) (2009) 77-90

24. Gros, C., Kaczor, G.: Semantic learning in autonomously active recurrent neural networks. Logic Journal of IGPL 18(5) (2010) 686-704

25. Linkerhand, M., Gros, C.: Generating functionals for autonomous latching dynamics in attractor relict networks. Scientific Reports 3 (2013)

26. Beer, R.D.: Dynamical approaches to cognitive science. Trends in cognitive sciences 4(3) (2000) 91-99

27. Deco, G., Rolls, E.T., Romo, R.: Synaptic dynamics and decision making. Proceedings of the National Academy of Sciences 107(16) (2010) 7545-7549 